\title{
Muslim Faith Leaders: De Facto Mental Health Providers and Key Allies in Dismantling Barriers Preventing British Muslims from Accessing Mental Health Care
}

\section{Sazan Meran}

School of Psychology, Faculty of Health and Medical Sciences, University of Surrey

\section{Oliver Mason}

School of Psychology, Faculty of Health and Medical Sciences, University of Surrey

\section{Author Note}

Correspondence concerning this article should be addressed to: Sazan Meran, 301 Pyrene House, Clayponds Lane, Brentford, Middlesex, TW8 0GR. Email: sazan.meran@gmail.com

\section{Abstract}

British Muslims habitually respond to the onset of mental ill-health by turning to their faith leaders. How Muslim faith leaders manage these encounters remains largely unexplored. In this study, 41 Muslim religious leaders in the UK completed vignette-based surveys depicting an individual meeting DSM-IV criteria for depression or schizophrenia. Participants were questioned regarding beliefs about etiology and treatment, counseling training and activity, and referral behavior; stigma levels were also measured. Muslim faith leaders were found to exhibit low stigma, provide substantial informal counseling, and routinely refer individuals to mainstream mental health services. They simultaneously embraced environ- 
mental, biological, and religious causes for mental illness. Muslim faith leaders emerge as potential allies in efforts to improve mental health outcomes for British Muslims, by challenging community stigma and collaborating with mental health professionals to deliver holistic care.

In 2005, the U.K. Department of Health published an action plan for delivering race equality in mental health care and found a significant need to provide culturally appropriate services to individuals from ethnic minorities, as well as address high levels of distrust of mental health professionals among these groups (Department of Health, 2005). With an estimated population of 2.7 million, British Muslims form the second largest religious group in the U.K. and are one of the most rapidly growing minority groups, with 1.2 million more people identifying as Muslim between 2001 and 2011, representing a jump from 3 to 5 percent of the overall population (Office for National Statistics [ONS], 2013). Muslims in the U.K. form a heterogeneous and ethnically diverse group, with 68 percent from a South Asian background, 10 percent reporting as Black (African/Caribbean or British), and 11 percent identifying as 'Other Ethnic Group' (ONS, 2013). British Muslims are among the worst affected by health inequalities, and have the highest reported rates of illness and disability of all minority groups (Hussain, 2009; Sheikh, 2007). In the current political climate, where Muslims are increasingly portrayed negatively (Ahmed \& Matthes, 2017), the mental health of this group is under particular threat (Sheridan, 2006; Ali, Milstein, \& Marzuk, 2005; Ali, Liu, \& Humedian, 2004). A study of Muslims living in Britain, France, and Germany found that perceived Islamophobia predicted higher levels of psychological distress and perceived stress (Kunst, Sam, \& Ulleberg, 2013), and evidence suggests that South Asian populations in the U.K. have a heightened risk of psychological morbidity (Fazil \& Cochrane, 2003). Minority ethnic groups face barriers to accessing mental health services, due in part to mistrust of mental healthcare professionals and stigma within communities (Bowl, 2007; Williams, Turpin, \& Hardy, 2006; Keating \& Robertson, 2004; NIMHE, 2004). A survey of 152 members of a Pakistani Muslim community by mental health charity Rethink Mental Illness (2007) found a consistent lack of uptake of mental health services. There is evidence that South Asian Muslims in particular are less likely to access mental health services than all other groups (Sheikh \& Furnham, 2000). Services may also lack knowledge or understanding in approaching these groups, and are therefore commonly viewed as irrelevant (Weatherhead \& Daiches, 2010; Bhui \& Morgan, 2007; Ali et al., 2004).

Muslims habitually turn to faith leaders at times of emotional and psychiatric difficulty (Dein, 2013; Al-Krenawi, Graham, Dean, \& Eltaiba, 2004; McCabe \& Priebe, 2004; Cinnirella \& Loewenthal, 1999). Barn and Sidhu (2005) interviewed 54 Muslim Bangladeshi women who identified imams and tradi- 
tional healers as their main source of support in terms of seeking healthcare. Similarly, Dein, Alexander, and Napier (2008) report that a sample of Bangladeshi Muslims in East London turned to imams and traditional faith healers before seeing medical professionals. Another survey of 111 Muslims found that a majority believed in supernatural causes of physical and mental illness (including jinn possession and black magic), and 54 percent believed treatment should be sought from both doctors and religious figures simultaneously (Khalifa, Hardie, Latif, Jamil, \& Walker, 2011). In an observational study of 123 patients attending an Early Intervention Service for Psychosis, Singh and colleagues (2015) found black and Asian patients were more likely to pursue help from faith organizations, although this did not result in their delayed presentation to mental health services.

\section{Imams: De Facto Mental Health Providers}

The traditional role of an imam is to lead prayer, conduct religious ceremonies, deliver sermons, and provide spiritual and religious guidance (Haddad \& Lummis, 1987). The imam plays a central role in the communal and spiritual life of Muslims and has a great deal of trust and respect placed in them (Siddiqui, 2004). Since Sunni Islam does not have a formalized clergy system, most mosques do not operate with standardized religious hierarchy (Maqsood, 2008; Boender, 1999), and it can be difficult to clearly identify the providers of support in an Islamic context. There are a broad range of religious teachers, spiritual guides, and scholars based in mosques, community centers, and educational establishments, any of whom may be approached by members of the Muslim community struggling with psychological difficulties (Pilkingon, Msetfi, \& Watson, 2011). All of these are encompassed here in the category 'Muslim faith leaders'. Faith leaders may perform Ruqya, an Islamic prayer modality that uses recitation of Quran verses or supplications of the Prophet $\mathrm{Mu}$ hammad for healing (York, Perez-Chisti, Lewis, \& Yucel, 2011).

Examining pastoral care provided within different faith groups, Leavey (2008) interviewed seven Muslim religious leaders who felt they were the first port of call for individuals in their community with psychiatric problems, more so than Christian and Jewish clergy. Across faiths, clergy are frequently frontline responders for congregants facing the onset of mental illness, and serve to facilitate these individuals' access to a broader network of mental health services (Larson et al., 1988; Piedmont, 1968). This role is particularly significant in minority ethnic communities, given the context of high disparities in access to healthcare (Ali et al., 2005; Young, Griffith, \& Williams, 2003).

Despite the apparently central role played by Muslim faith leaders in the provision of support to individuals with psychological difficulties, highlighted 
by patients and religious leaders alike, there has been limited exploration of their role. What studies do exist in a U.K. context are qualitative in nature, utilizing a very small sample. For instance, Rashid, Copello, and Birchwood (2012) examined the views of eight Muslim faith leaders, including four imams, two shaykhs, one mullah, and one female religious teacher, on psychosis and substance misuse. The religious leadership simultaneously held medical and religious beliefs about the causes of mental illness, deploying both within the same narrative and finding no conflict between the two. Participants at times spoke about schizophrenia as having a biomedical etiology, requiring psychiatric intervention, and at other times stated they believed psychotic symptoms were caused by jinn possession. Similarly, Ally and Laher (2007) interviewed six Muslim faith healers in South Africa about the perceived causes of mental illness; this was sometimes held to have environmental or biological causes (stress, trauma, or chemical imbalances in the brain), although spiritual problems could present as mental ill-health. The healers claimed the ability to distinguish between spiritual illness with a supernatural cause, such as black magic or jinn, and mental illness. In another qualitative UK-based study, Watts, Murray, and Pilkington (2014) performed a phenomenological analysis of the experiences of six imams, all of whom saw providing counseling and pastoral care as a core part of their role and acted in a manner analogous to central diagnostic practitioners: Directing those with medical ailments to doctors, and those with jinn difficulties to a spiritual expert. Imams reflected that while they made referrals to medical services, a reciprocal relationship did not exist.

A small number of quantitative studies have been conducted in the U.S. on the role played by imams in the mental health of Muslims. Ali and colleagues (2005) surveyed 62 imams across the U.S., and found that 95 percent spent a significant amount of time each week counseling congregants with psychiatric difficulties. However, imams were less likely than clergy from other faith groups to have formal counseling training. In a second study, Ali and Milstein (2012) utilized a vignette-based survey depicting an individual exhibiting symptoms of depression to assess imams' beliefs about the etiology of mental illness and helpful interventions, as well as their referral practices and counseling experience. Their principal finding was that imams recognized the severity of serious mental illness and the necessity of some type of intervention in order to attain recovery. Imams were most willing to refer congregants to mental health professionals while continuing to provide counseling themselves. However, although imams recognized the validity of mental health interventions alongside religious interventions, actual referrals to mental health professionals were low. Abu-Ras, Gheith, and Cournos (2008) conducted a cross-sectional survey of 22 imams and 102 Muslim worshippers in 22 mosques in New York City, and found that members of the Muslim community most frequently sought support for mental health issues from imams. The majority of imams had not 
received any formal training in Western psychotherapy interventions, yet they nevertheless played a central role in promoting congregants' mental wellbeing through unstructured psychotherapy interventions.

\section{Perceptions of Illness Severity: Depression vs Schizophrenia}

While Ali and Milstein (2012) used a depression vignette in their survey of US-based imams, this study sought responses of Muslim faith leaders to vignettes of individuals with symptoms of both depression and schizophrenia. Evidence suggests that both the general public and religious groups have differing perceptions of individuals suffering from schizophrenia and depression, viewing those with schizophrenia as more dangerous (Pescosolido et al., 2010). This remains true even for individuals reporting familiarity with schizophrenia (Crisp, Gelder, Rix, Meltzer, \& Rowlands, 2000). In a study including several religious groups in Britain, Cinnirella and Loewenthal (1999) found that participants in all groups felt schizophrenia was more serious and more likely to have an organic cause than depression. In contrast, depression was more likely to be viewed as resulting from a religious failing on the part of the patient.

\section{Beliefs about Etiology and Treatment}

Research shows that beliefs about what causes psychiatric illness affect the likelihood of a person accessing support from mental health services (Hill \& Bale, 1980). Explanatory models - the set of assumptions held about the causes of ill-health - are profoundly different across cultures and influence the mode of help sought by an individual when unwell (Lynch \& Medin, 2006). Consequently, this study asks whether Muslim faith leaders attribute mental illness to environmental, biological, or religious causes, and how these beliefs about etiology impact beliefs about appropriate treatment responses, as well as referral practices to mainstream mental health services.

\section{Mental Health Stigma}

Several ethnic and cultural minority groups have been found to stigmatize those with mental health difficulties to different degrees (Rao, Feinglass, \& Corrigan, 2007), and high levels of mental health stigma have been specifically found within Muslim communities (Cifti, Jones, \& Corrigan, 2012; Tabassum, Macaskill, \& Ahmad, 2000; Cinnirella \& Loewenthal, 1999). However, Muslim faith leaders are likely to have considerably more contact with congregants in 
mental distress or with mental health problems and the degree of social distance they desire is unknown. It is plausible that they hold very different perceptions of mental illness than British Muslims in general, and have less stigmatized views. This is because successive studies have found that individuals with greater experience of mental illness are less prone to endorse stereotypes of dangerousness or desire social distance (Angermayer, Matschinger, \& Corrigan, 2004; Corrigan, Edwards, Green, Diwan, \& Penn, 2001; Penn et al., 1994).

\section{Aims and Hypotheses}

Muslim faith leaders in the U.K. are seemingly playing significant roles as front-line responders to individuals experiencing psychological difficulties; This study aims to broaden our understanding of their beliefs and behaviors when providing this mental health support. Due to evidence that British Muslims facing mental illness routinely turn to their religious figures, it is hypothesized that Muslim faith leaders are providing substantial informal counseling in British Muslim communities. However, given findings of limited formal counseling training among imams in the U.S. (Ali et al., 2005), similarly low levels are anticipated here. Furthermore, with evidence of an inverse relationship between contact and stigma, Muslim faith leaders reporting greater familiarity with mental illness are hypothesized to exhibit less stigma. Nevertheless, based on prior vignette studies, it is expected that participants will desire greater social distance from those with schizophrenia, perceiving the patient to be more dangerous and the illness more severe than depression. Finally, it is hypothesized that Muslim faith leaders attributing mental illness to spiritual causes will be more likely to view religious interventions as helpful, and less inclined to direct individuals to seek medical help.

\section{Method}

\section{Participants}

Forty-one UK-based Muslim faith leaders completed the survey, including imams, shaykhs, and both male and female graduates of religious leadership courses. Of the 41 participants, 37 were male (90\%) and 4 were female (10\%). Just over one-third of participants were between the ages of 35 and 44, and one-third between 45 and 54 years old (15 participants each); the remaining third was split between those reporting to be $25-34$ years of age (6 participants, $15 \%$ of the sample), three (7\%) between $55-64$, one each $(2 \%)$ at the two ends of the age spectrum (between 18-24 years, and between 65-74 years old). Nine 
respondents (22\%) reported their ethnicity as Asian/Asian British (Pakistani), six (15\%) as Asian/Asian British (Bangladeshi), six (15\%) as Black/Black British (African), five (12\%) as Asian/Asian British (Indian), four (10\%) as Arab, two (5\%) as Black/Black British (Caribbean), one (2\%) as White (Other), one (2\%) as Mixed (Other), and seven (17\%) as Other Ethnic Group, including two Iranians, one South-east Asian, one Kosovan, and one Albanian.

Regarding tenure in the U.K., 5 were born there (12\%), 15 (37\%) had lived in England for more than 20 years, 12 (29\%) for 11-20 years, six (15\%) for 6-10 years, and three (7\%) for less than five years. Nineteen (46\%) participants had a postgraduate degree, $10(24 \%)$ had an undergraduate degree, eight (20\%) had a doctoral degree, three (7\%) had completed college/sixth form and one participant did not report the highest level of education they had completed. Thirtyeight (93\%) individuals reported receiving a traditional Islamic education.

\section{Measures}

Participants were asked to complete a survey taking approximately 20 minutes. Demographic information was collected, including gender, age, ethnicity, number of years spent as Muslim faith leader, education levels, and number of years spent living in the U.K.

As they were recruited to the study, individuals were alternately allocated to one of two vignette conditions, adapted from the mental health modules of the 1996 and 2006 General Social Survey (Pescosolido, 2010). The first vignette depicted an individual named Ahmad who met the Diagnostic and Statistical Manual of Mental Disorders' criteria for depression (4th ed.; DSM-IV; American Psychological Association, 1994), and the second portrayed an individual named Zaid who met the DSM-IV criteria for schizophrenia. The vignettes were adapted to describe individuals who had a religious upbringing and whose families attributed the difficulties they were experiencing to black magic (sihr) or jinn, in an attempt to emulate the type of individual a Muslim faith leader may realistically be asked to support. They do not contain any medical diagnoses, to simulate the experience of faith leaders in the community, who are likely to see a person presenting with problematic behaviors but not medical labels or history. Twenty participants were given the schizophrenia vignette, and the remaining twenty-one were given the depression vignette, using alternating allocation. After reading the vignettes, participants were asked whether they understood the paragraph. Following this, participants were asked questions in seven areas:

Stigma. Measures for social distance and perceptions of dangerousness were taken from Pescosolido and colleagues (2010). Participants were asked how willing they would be for the individual depicted in the vignette to live next door; socialize with them for an evening; be their friend; work closely with 
them on a job; and marry their children. "Definitely unwilling" and "probably unwilling" were coded 1 (i.e. stigmatizing) and "probably willing", "definitely willing", and "do not know" were coded 0 . The internal reliability of the scale was acceptable, with Cronbach's $\alpha$ at 0.75 . An overall score for social distance was created by adding together the scores from each of the above five social distance categories, yielding scores between 0 (least stigmatizing) and 5 (most stigmatizing) for each participant.

The second measure asked participants how likely it was that the person described in the vignette would "do something violent toward other people" and/or "do something violent toward himself". Responses of "very likely" and "somewhat likely" were coded 1; responses of "not very likely", "not at all likely", and "do not know" were coded 0.

Familiarity. Familiarity was measured using Holmes and colleagues' (1999) Level of Contact Report. This outlines 12 situations, listed in Table 1, that range in closeness of contact with mentally ill individuals. These situations vary from most intimate ("I have a mental illness"), to medium intimacy ("I have worked with a person who had a severe mental illness at my place of employment"), to least intimate ("I have never observed a person I was aware had a mental illness"). Participants were asked to indicate all of the situations across the 12 items that they had ever experienced. The situations were ranked in terms of intimacy by three experts in mental illness; the mean of rank-order correlations summarizing inter-rater reliability was 0.83 . This rank order was subsequently validated by a sample of 100 participants (Holmes et al., 1999).

The index for familiarity in this study was the rank score of the most intimate situation indicated by the participant. For example, a participant who

Table 1. Level-of-contact Report

\begin{tabular}{ll}
\hline Score & \multicolumn{1}{c}{ Description } \\
\hline 3 & $\begin{array}{c}\text { I have watched a movie or television show in which a character depicted a } \\
\text { person with mental illness. } \\
\text { My job involves providing services/treatment for persons with a severe mental } \\
\text { illness. }\end{array}$ \\
$\begin{array}{l}\text { I have observed, in passing, a person I believe may have had a mental illness. } \\
5\end{array}$ & I have observed persons with a mental illness on a frequent basis. \\
12 & I have a mental illness. \\
6 & I have worked with a person who had a mental illness at my place of \\
1 & employment. \\
7 & I have never observed a person that I was aware had a mental illness. \\
9 & My job includes providing services to persons with a mental illness. \\
10 & I friend of the family has a mental illness. \\
4 & I have watched a documentary on the television about mental illness. \\
11 & I live with a person who has a mental illness.
\end{tabular}


chose two situations from the list - "I have worked with a person who had a mental illness at my place of employment" (score $=6$ ) and "I have a relative who has a mental illness" (score $=10$ ) would be designated a score of 10 because "I have a relative who has a mental illness" is the more intimate situation.

Severity of Illness. As in Ali and Milstein's (2012) study, participants were asked how serious they considered the problem faced by the individual in the vignette to be, answering on a 4 -point Likert scale ( $1=$ very serious; $2=$ somewhat serious; $3=$ not very serious; $4=$ not at all serious). Participants were also asked how likely it was that the individual's situation would improve on its own, answering on a 5 -point Likert scale ( $1=$ very likely; 2 = somewhat likely; $3=$ not very likely; $4=$ not at all likely; $5=$ do not know).

Etiology. Participants were asked how likely it was that Ahmad/Zaid's situation was caused by "a religious or spiritual problem", "the way he was raised", "black magic (sihr) or jinn", "stressful circumstances in his life", "a chemical imbalance in the brain" and "a genetic or inherited problem". Questions were not mutually exclusive, and participants could endorse multiple attributions. Participants responded on a 5-point Likert scale (very likely $=1$; somewhat likely $=2$; not very likely $=3$; not at all likely $=4$; do not know $=$ 5). Responses attributing depression or schizophrenia to "black magic (sihr) or jinn" and/or "a religious or spiritual problem" were coded 1 for belief in religious causes, and otherwise coded 0 . Likewise, participants were coded 1 for belief in environmental causes if they chose "stressful circumstances in his life" and/or "the way he was raised", and coded 1 for belief in biological causes if they attributed Ahmad or Zaid's difficulties to "a chemical imbalance in the brain" and/or "a genetic or inherited problem". In both cases, participants were otherwise coded 0 . This measure was adapted from Ali and Milstein (2012) to explicitly ask about beliefs in sihr and jinn. Despite the absence of formal evidence of validity, the measure was shown to perform adequately in this study.

Treatment. Participants were asked how helpful certain actions would be for Ahmad/Zaid, including "talking to family/friends", "talking to an imam/ shaykh", "talking to a therapist/counselor", "going to a raaqi", "going to his GP", "seeing a psychiatrist", "reading Quran, praying, fasting, and/or attending mosque more" and "taking psychiatric medication". Questions were not mutually exclusive, and participants could endorse multiple helpful actions on a 5-point Likert scale (very helpful = 1; somewhat helpful = 2; somewhat unhelp$\mathrm{ful}=3$; very unhelpful $=4$; do not know $=5$ ). This measure was also adapted from Ali and Milstein's (2012) study of U.S. imams, to explicitly ask about attitudes toward medical and spiritual interventions for psychological distress. Once again, despite the absence of formal evidence of validity, the measure was shown to perform adequately in this study.

Counseling Activity and Referral Behavior. Participants were asked how 
many hours per week they typically spent counseling individuals with psychological difficulties or mental health problems, selecting from "none", "1-5 hours", "6-20 hours" and "Over 20 hours". Participants recorded whether for any of the individuals they had counseled within the past year, they had either consulted with or referred to a GP, social worker, imam/shaykh familiar with psychotherapy/mental illness, psychiatrist or psychologist, community mental health team/nurse, psychiatric hospital or A\&E, or a therapist. Participants answered either "yes", "no" or "not sure".

Counseling Education and Training. Participants were asked whether they had completed any "college psychology courses", "personal reading in counseling", "courses in psychology/mental health at Islamic school", "other training or counseling experiences", "consultation/supervision with a mental health professional", "counseling classes from an Islamic organization", "Clinical Pastoral Training", and "courses on mental illness", selecting either "yes", "no" or "not sure". Participants were asked whether they felt the training they had received was adequate to recognize mental illness, again answering "yes", "no" or "not sure". Finally, participants were asked if there was anything further they wished to add following the completion of the survey, and were given the opportunity to respond freely.

\section{Recruitment}

Participants were recruited through mosques, Islamic community organizations and educational establishments, and using snowballing techniques. Only Muslim faith leaders based in the U.K. were included in the study. Due to differing levels of reading comprehension in the English language, a number of participants requested the survey be read aloud and their responses recorded by the author, either face-to-face or over the telephone. This request was accommodated whenever required. There were no instances when interpretation into the participant's native language was needed.

\section{Ethical considerations}

Formal ethical approval for the study was obtained from the Faculty of Health and Medical Sciences Ethics Committee at the University of Surrey. Informed verbal consent was obtained prior to beginning the survey. All participants were assured that their involvement was voluntary, that they could refuse to answer any question, or withdraw entirely without explanation, and were provided with details of the researcher and supervisor. 


\section{Results}

As several of the variables are categorical, and those using Likert scales did not approximate normal distributions, parametric statistical analysis was precluded. As a result, non-parametric statistics were used. Spearman's Rho correlation was used to test associations within the data. Differences between the depression and schizophrenia vignette conditions were analysed using a Mann Whitney test.

Views of schizophrenia: Social Distance, perception of danger and familiarity

The hypothesis that stigma would be greater toward those with schizophrenia was not supported as there was no significant difference in desire for social distance between the schizophrenia and depression conditions. Participants' responses were therefore collapsed across conditions: Most (36, 88\%) were willing to live next door to Ahmad/Zaid, and nearly all (40,98\%) participants were willing to spend an evening socializing with Ahmad/Zaid and to have Ahmad/ Zaid as a friend. Most $(33,81 \%)$ were willing to work closely with Ahmad/Zaid on a job. Finally, over half $(22,54 \%)$ were willing to have their children marry someone like Ahmad/Zaid.

However, consistent with the hypothesis, nearly twice as many participants believed the character with schizophrenia $(14,70 \%)$ would be violent to others than did so for depression (8, 38\%: $U=143.0, z=-2.02, p<.05)$. This was a small-to-medium effect (Cohen, 1988). There was no difference in beliefs about self-harm: 17 (81\%) participants in the depression vignette condition believed it was likely that Ahmad would be violent toward himself, and 17 (85\%) participants in the schizophrenia condition believed Zaid would harm himself. Contradicting the hypothesis that those with higher familiarity scores would exhibit lower stigma levels, no significant correlation was found between familiarity and stigma in this sample.

Severity of Illness

Muslim faith leaders demonstrated their ability to recognize the severity of mental illness as depicted in the vignettes, and, in the case of the individual exhibiting schizophrenia symptoms, the need for intervention. Contradicting the hypothesis that schizophrenia would be considered a more serious mental illness than depression, there was no significant difference in responses regarding the severity of the problem faced by the individuals depicted in either the schizophrenia or depression vignettes. Findings were therefore collapsed across conditions: The large majority $(36,88 \%)$ identified the individual's difficulties as serious. 
However, recognition of the importance of intervention for schizophrenia sufferers does support the hypothesis that this psychiatric illness would be perceived as the more critical. Nine (42\%) participants in the depression vignette condition believed it likely that Ahmad's situation would improve on its own. Eleven (52\%) believed it unlikely that his situation would improve spontaneously, and one participant said they did not know. In contrast, two (10\%) participants in the schizophrenia condition believed it likely that Zaid's situation would improve on its own, while 18 (90\%) believed it was unlikely. A Mann-Whitney test indicated that participants in the depression vignette condition were significantly more likely to think the individual's situation would improve without intervention than participants in the schizophrenia vignette condition $(U=150.00, z=-1.98, p<.05, r=-0.31)$. The effect size indicates a small-to-medium effect (Cohen, 1988).

\section{Beliefs about Etiology and Treatment}

There was no significant difference in attributional beliefs in response to the schizophrenia and depression vignettes, and results were therefore combined across conditions. All participants endorsed more than one cause at the same time: Almost all $(39,95.10 \%)$ attributed Ahmad/Zaid's difficulties to an environmental cause, over two-thirds $(29,71 \%)$ to a biological cause, and over half $(24,59 \%)$ to a religious cause. No participants in either vignette condition attributed the difficulties faced by Ahmad/Zaid solely to a religious cause. There were no significant correlations between attributions of Ahmad/Zaid's difficulties to religious causes and either environmental or biological causes, and so participants who believed the difficulties described in the vignette may have a religious cause were no less likely to simultaneously believe in environmental or biological causes.

There was also no significant difference in treatment beliefs between the schizophrenia and depression conditions. All participants reported that either one or more form of Islamic intervention would be helpful: the great majority $(38,93 \%)$ said speaking to an imam would be helpful and the same number recommended reading Qur'an, praying, fasting, and/or attending the mosque more, while just over half $(24,59 \%)$ believed seeing a raaqi would benefit Ah$\mathrm{mad} /$ Zaid. However, a similarly high percentage (37, 90\%) believed medical intervention in the form of seeing a psychiatrist or taking psychiatric medication would be beneficial.

As hypothesized, there was a positive correlation between attributing mental illness to spiritual causes and support for religious interventions: Participants who believed the mental health difficulties described in the vignettes were likely caused by black magic (sihr) or jinn were more prone to state that seeing a raaqi would be helpful to Ahmad/Zaid ( $r s=.42, p<.01)$. However, 
Muslim faith leaders holding religious attributional beliefs were no less likely to believe medical interventions would be helpful ( $r s=.05, p=.74)$.

\section{Counseling Activity and Referral Behavior}

Nearly two-thirds $(26,63 \%)$ of participants reported counseling individuals with mental health problems on a weekly basis, supporting the hypothesis that Muslim faith leaders are playing a substantial role in responding to individuals with psychological difficulties. Around half $(21,51 \%)$ said they spent between 1 and 5 hours per week doing so. Only three (7\%) spent 6-20 hours per week counseling, and two (5\%) spent over 20 hours counseling per week. None had referred individuals they had seen in the past year solely to another imam or shaykh familiar with psychotherapy. Thirty-five (85\%) reported referring individuals to a mixture of services, as outlined in Table 2. There was no relationship between referral behavior and beliefs about etiology, contrary to the prediction that Muslim faith leaders who attribute mental illness to spiritual causes would be less likely to refer people to mental health services ( $r$ s $=-.07$, $p=.67)$.

\section{Counseling Education and Training}

Nearly three-quarters of total participants $(30,73 \%)$ reported receiving some form of training in counseling or mental illness, contradicting the hypothesis that this group would have low levels of formal training, and many had received multiple experiences (see Table 3). Just three (7\%) reported receiving training solely via Islamic organizations, perhaps supplemented with personal reading. Eight (20\%) faith leaders said they had not received formal training of any kind, but of these, five (12\%) said they had done personal reading in counseling. Under half $(17,42 \%)$ faith leaders said they felt their training was adequate to recognize mental illness, while a third $(14,34 \%)$ felt their training was not adequate, and a quarter $(10,24 \%)$ of participants were not sure.

Table 2. Referral/Consultation Practices of Muslim Faith Leaders in the Past Year

\begin{tabular}{lcc}
\hline Type of Referral/Consultation & Frequency & Percentage \\
\hline General Practitioner (GP) & 26 & 63 \\
Social Worker & 12 & 29 \\
Imam/Shaykh familiar with psychotherapy/ & 15 & 37 \\
$\quad$ mental illness & & 56 \\
Psychiatrist/Psychologist & 23 & 17 \\
Community mental health team/nurse & 7 & 29 \\
Psychiatric hospital/A\&E & 12 & 42 \\
Therapist & 17 & \\
\hline
\end{tabular}


Table 3. Counseling Training Experiences of Muslim Faith Leaders

\begin{tabular}{lcc}
\hline Type of training & Frequency & Percentage \\
\hline College psychology course & 10 & 24 \\
Personal reading in counseling & 33 & 81 \\
Courses in psychology/mental health at Islamic & 13 & 32 \\
$\quad$ School & 29 & 71 \\
Other training or counseling experiences & 7 & 17 \\
Consultation/supervision with a mental health & & \\
$\quad$ professional & 15 & 37 \\
Counseling classes from an Islamic organization & 6 & 15 \\
Clinical Pastoral Training & 14 & 34 \\
Courses on mental illness & & \\
\hline
\end{tabular}

\section{Discussion}

This study is the first attempt to describe and quantify UK-based Muslim faith leaders' responses to individuals presenting to them with symptoms of mental illness. It has confirmed that these religious leaders are seen by many British Muslims as valid providers of treatment for psychological difficulties, revealed the extent of the informal counseling they deliver, and indicated relatively low mental health stigma in this group.

It was hypothesized that due to Muslim faith leaders' frequent contact with those with psychological difficulties, borne of the substantial counseling role they play in the community, they would have high familiarity with and therefore exhibit relatively lower stigmatizing attitudes toward mentally ill individuals. This hypothesis was in line with evidence indicating that contact increases familiarity, which is linked with reduced stigma in turn (Angermeyer et al., 2004; Corrigan et al., 2001; Penn et al., 1994).

While no association was found between familiarity and stigma in this study, Muslim faith leaders did express comparatively less stigma toward individuals with mental illness than has been found in the broader Muslim community. On measures of social distance, nearly all participants were willing to live next door to or spend an evening socializing with the individuals depicted in the vignettes, or to have them as a friend. Over two-thirds were willing to work closely with Ahmad or Zaid on a job, while over half were willing to have their children marry someone like them. These findings are strikingly different from those reported by Tabassum and colleagues (2000) in their study of 45 British Pakistani Muslims. None in that sample would consider marriage with an individual with mental illness, while less than a quarter would consider a close relationship, and less than half would be prepared to socialize with such a person. In addition, numerous descriptive studies have highlighted that mental health stigma within Muslim communities constitutes a particu- 
larly troublesome barrier to accessing services, more so than in other religious groups (Leavey, 2008; Bradby et al., 2007; Gilbert, Gilbert, \& Sanghera, 2004).

It is unclear why no correlation was found between familiarity and stigma levels in this study given the established link between these two factors in the literature. Perhaps the sample size was too small to detect a relationship, or there may be a third, unknown factor responsible for lower desire for social distance among Muslim faith leaders. This is a worthwhile area for further investigation given the near absence of literature exploring stigma levels in this group.

While Muslim faith leaders scored relatively better on measures of social distance, over two-thirds believed it likely that the individual depicted with schizophrenia was likely to commit acts of violence toward others. This aligns with successive studies illustrating that schizophrenia patients are perceived as dangerous (Pescosolido et al., 2010; Crisp, Gelder, Goddard, \& Meltzer, 2005). Although those with schizophrenia are indeed at an elevated risk of perpetrating acts of violence (Wallace, Mullen, \& Burgess, 2004), public fears substantially inflate this risk (Crisp et al., 2000; Steadman et al., 1998). Thus, there remains work to do in adjusting misconceptions of those with schizophrenia among Muslim faith leaders.

Why do stigma levels among Muslim faith leaders matter? As hypothesized, these leaders play a considerable role in responding to the needs of individuals in their communities with mental health problems. Nearly twothirds of participants reported spending time each week counseling those with psychological difficulties, with over half reporting spending between one to five hours weekly, and the remainder spending between 6 and over 20 hours per week. This quantifies findings in earlier qualitative UK-based studies in which imams stated that providing help to community members with mental illness was central to their role (Watts et al., 2014; Leavey, 2008), and British Muslims expressed a strong tendency to first approach religious leaders when experiencing psychological distress (Dein, 2013; Barn \& Sidhu, 2005; McCabe \& Priebe, 2004). Consequently, Muslim faith leaders' attitudes to mental illness set the context in which many individuals in their community react to the onset of psychiatric ill-health. Such attitudes can translate into acceptance or rejection; prompt or delayed help-seeking; empathy or fear.

So why have the attitudes of Muslim religious leaders not already translated into reduced stigma toward the mentally ill in the Muslim community? While imams clearly play a significant role in providing informal counseling and signposting to mental health services, the first intervention study challenging mental health stigma among Muslims in the U.K. supported the power of social contact with an individual with lived experience of mental illness to reduce mental health stigma in this group (Hankir et al., 2017). This suggests that partnerships between Muslim faith leaders, experts by lived experience and 
mainstream mental health professionals may hold the key to reducing mental health stigma in British Muslim communities.

Muslim faith leaders are also crucial allies in any attempts increase trust in mental health professionals. The results revealed that participants frequently directed individuals with psychological difficulties to seek help from mainstream healthcare providers, with the vast majority making such referrals to other agencies and services in the past year. Nearly two-thirds had directed individuals to see their GP and over half had recommended consulting a psychiatrist or psychologist. This represents higher referral rates than reported in a comparative study of US-based imams, where less than half had referred congregants to a primary care physician, or a psychiatrist or psychologist (Ali \& Milstein, 2012). In line with the study's hypotheses, Muslim faith leaders who attributed mental illness to spiritual causes were more likely to view religious interventions (in the form of seeing a raaqi) as helpful. However, this was not associated with an eschewing of biological or environmental causes, the rejection of medical interventions, or, contrary our hypothesis, a reduced tendency to refer congregants to mainstream mental health providers.

Muslim faith leaders appear to be actively encouraging British Muslim communities to overcome their aversion to mental health services. Given the absence of formal referral routes between these faith leaders and health services, it is not known whether individuals do in fact act on these recommendations, highlighting another important avenue for future research. However, evidence of low uptake of services among British Muslims (Rethink, 2007; Williams et al., 2006), perhaps demonstrates that even the recommendations of highly respected religious figures struggle to overcome the stigma associated with utilizing formal mental health support, or the belief that mental health services are irrelevant (Weatherhead \& Daiches, 2010; Sheikh \& Gatrad, 2009). Hence, tackling stigma alongside delivering religiously literate mental healthcare may hold the key to positively engaging British Muslims with mental health services.

Mental health professionals should consider Muslim faith leaders an invaluable resource in any effort to provide effective care to British Muslim patients. Broadly, Muslim faith leaders can educate mental health professionals about the importance of religion to an individual's healing and recovery. In turn, health professionals can teach Muslim faith leaders to recognize and understand mental illness from a medical viewpoint. However, the practicalities of collaborative working when an individual wants to pursue both an Islamic and medical approach to receiving mental health support require greater formulation: In what instances do these approaches positively reinforce one another, when can they simply co-exist, and when is there irresolvable incompatibility? A principal area of negotiation will concern beliefs regarding the etiology of mental illness. 
Our findings reveal that Muslim faith leaders most frequently ascribed mental illness to environmental reasons, followed by biological and then religious causes. However, they often embraced all three concurrently. This apparent syncretism between seemingly conflicting explanatory frameworks of mental illness has been evidenced in earlier studies of both Muslim lay persons and religious figures (Dein, 2013; Rashid et al., 2012; Khalifa et al. 2011). Muslim faith leaders seem open to a wide range of possible etiologies and so may variously suggest medical, psychological/emotional, and spiritual sources of support for the same individual. This may explain why the belief in spiritual causes did not translate to a rejection of medical interventions, as had been hypothesized.

Can mental health professionals in turn make room for religion and spirituality? Research shows that mental health patients are strongly averse to talking about their religious beliefs and behaviors for fear of facing cultural insensitivity, or even being branded deluded and hospitalized involuntarily (Dein, 2013; De Sousa, 2007). This is despite research indicating that respect for and understanding of a person's religious beliefs is essential in developing meaningful therapeutic relationships (Weatherhead \& Daiches, 2010). Such respect and understanding may be realized through the proposed partnership between mental health professionals and Muslim faith leaders. A tendency for psychiatrists to resist working with faith-based organizations has been noted (DuràVilà, Hagger, Dein, \& Leavey, 2011), and it is true that the resolution of conflicting beliefs and treatment modalities may be problematic (Leavey, 2010). While the case cannot be made here that such antagonisms are surmountable, the implementation of such collaborations may improve the mental health care of a marginalized and vulnerable, but rapidly growing minority community in the U.K. One promising example is Mir and colleagues' (2015) incorporation of Islamic teachings in an adapted behavioral activation intervention for depression, which was found to be feasible to implement in practice, and acceptable to most patients, therapists, and referral agencies.

Enhancing the mental health training of Muslim faith leaders may aid in the development of these working relationships. It was predicted that participants would have low levels of formal counseling training, as was found in a sample of American imams (Ali et al., 2005). The results suggest that while levels were not low, more - and perhaps better quality - training is required. Over two thirds had received some form of training in counseling or mental illness. Despite this, a majority did not believe their training was adequate to recognize mental illness. In addition, while most Muslim faith leaders surveyed were able to recognize the severity of the mental illnesses depicted in the vignettes, over a third believed Ahmad's depression was likely to resolve spontaneously without intervention. Significantly, only six respondents had Clinical Pastoral Training. Improving uptake of this training among Muslim faith leaders may facilitate 
working partnerships with mental health professionals, since it provides a way to approach mental healthcare as part of a multidisciplinary team (Vandecreek \& Lucas, 2001). Improved mental health training is also important since imams are in a unique position to help boost mental health literacy among British Muslims, and to aid in the recognition, management, or prevention of mental illnesses (Jorm et al., 1997).

The results of this study should be interpreted within the context of several limitations. The sample size used here is relatively small, which is an important limitation in a group as diverse as British Muslims. While the vignettes used were an attempt to replicate the types of encounters Muslim faith leaders may experience day-to-day, as well as provide a vivid depiction of someone with mental illness, they cannot truly mimic or evoke real-life responses, possibly compromising the ecological validity of our findings. Additionally, social desirability bias may have led participants to report more positive attitudes toward those with mental illness, or be less inclined to admit to beliefs in spiritual causes and remedies. This tendency to respond in a manner deemed socially acceptable may be a particular problem in a community currently under heavy and unwelcome scrutiny (Chakraborti, 2013; Awan, 2012, Nickels, Thomas, Hickman, \& Silvestri, 2012), and highlights the importance of fostering trust when researching British Muslims.

This study represents an important step in building a picture of the role played by Muslim faith leaders in responding to the mental health needs of their communities. Further research is vital given the dearth of empirical research on this topic in the U.K., and some possible future directions have already been noted. Given the rich potential for partnership between Muslim faith leaders and mental health professionals, there is a need to develop, trial, and evaluate collaborative efforts. In addition, it appears that Muslim faith leaders believe it possible to distinguish between mental and spiritual problems, but more needs to be understood about how this diagnostic discrimination is made, and what consequences there are for the individual in need of support. Finally, it would be interesting to investigate the satisfaction levels of British Muslims who have turned to faith leaders for mental health support, to better understand their expectations when approaching religious figures , and to determine whether they felt their needs had been adequately met.

\section{References}

Abu-Ras, W., Gheith, A., \& Cournos, F. (2008). The Imams Role in Mental Health Promotion: A Study at 22 Mosques in New York Citys Muslim Community. Journal of Muslim Mental Health,3(2), 155-176. https://doi.org/10.1080/15564900802487576

Ahmed, S., \& Matthes, J. (2017). Media representation of Muslims and Islam from 2000 
to 2015: A meta-analysis. International Communication Gazette, 79(3), 219-244. https://doi.org/10.1177/1748048516656305

Ali, O. M., \& Milstein, G. (2012). Mental Illness Recognition and Referral Practices Among Imams in the United States. Journal of Muslim Mental Health, 6(2). https:// doi.org/10.3998/jmmh.10381607.0006.202

Ali, O., Milstein, G., \& Marzuk, P. (2005). The Imam's role in meeting the counseling needs of Muslim communities in the United States. Psychiatric Services (Washington, D.C.), 56(2), 202-5. https://doi.org/10.1176/appi.ps.56.2.202

Ali, S. R., Liu, W. M., \& Humedian, M. (2004). Islam 101: Understanding the religion and therapy implications. Professional Psychology: Research and Practice, 35, 635642. https://doi.org/10.1037/0735-7028.35.6.635

Al-Krenawi, A., Graham, J. R., Dean, Y. Z., \& Eltaiba, N. (2004). Cross-national study of attitudes towards seeking professional help: Jordan, United Arab Emirates (UAE) and Arabs in Israel. International Journal of Social Psychiatry, 50(2), 102114. https://doi.org/10.1177/0020764004040957

Ally, Y., \& Laher, S. (2007). South African Muslim Faith Healers Perceptions of Mental Illness: Understanding, Aetiology and Treatment. Journal of Religion and Health,47(1), 45-56. https://doi.org/10.1007/s10943-007-9133-2

American Psychiatric Association. (1994). Diagnostic and statistical manual of mental disorders (4th ed.). Washington, DC: Author.

Angermeyer, M. C., Matschinger, H., \& Corrigan, P. W. (2004). Familiarity with mental illness and social distance from people with schizophrenia and major depression: Testing a model using data from a representative population survey. Schizophrenia Research,69(2-3), 175-182. https://doi.org/10.1016/S0920-9964(03)00186-5

Awan, I. (2012). "I Am a Muslim Not an Extremist": How the Prevent Strategy Has Constructed a "Suspect" Community. Politics \& Policy, 40(6), 1158-1185. https:// doi.org/10.1111/j.1747-1346.2012.00397.x

Barn, R., \& Sidhu, K. (2005). Understanding the Interconnections Between Ethnicity, Gender, Social Class and Health. Social Work in Health Care,39(1-2), 11-27. https://doi.org/10.1300/J010v39n01_03

Bhui, K., \& Morgan, N. (2007). Effective psychotherapy in a racially and culturally diverse society. Advances in Psychiatric Treatment, 13(3), 187-193. https://doi. org/10.1192/apt.bp.106.002295

Boender, W. (1999). Imams in the Netherlands: An impression. Centrum Voor Islam in Europa, 1(2), 23-28.

Bowl, R. (2007). The Need for Change in UK Mental Health Services: South Asian Service Users' Views. Ethnicity \& Health, 12(1), 1-19. https://doi. org/10.1080/13557850601002239

Chakraborti, N. (2013). Policing Muslim communities. In Policing Beyond Macpherson: Issues in Policing, Race and Society (pp. 107-127). Taylor and Francis.

Cinnirella, M., \& Loewenthal, K. (1999). Religious and ethnic group influences on beliefs about mental illness: A qualitative interview study. British Journal Of Medical Psychology, 72(4), 505-524. https://doi.org/10.1348/000711299160202

Cohen, J. (1988). Statistical power analysis for the social sciences (2nd ed.). Hillsdale, NJ7 Erlbaum.

Corrigan, P. W., Edwards, A. B., Green, A., Diwan, S. L., \& Penn, D. L. (2001). Prejudice, Social Distance, and Familiarity with Mental Illness. Schizophrenia Bulletin,27(2), 219-225. https://doi.org/10.1093/oxfordjournals.schbul.a006868 
Crisp, A., Gelder, M., Goddard, E., \& Meltzer, H. (2005). Stigmatization of people with mental illnesses: a follow-up study within the Changing Minds campaign of the Royal College of Psychiatrists. World Psychiatry, 4(2), 106-113.

Crisp, A., Gelder, M., Rix, S., Meltzer, H., \& Rowlands, O. (2000). Stigmatisation of people with mental illnesses. The British Journal of Psychiatry: The Journal of Mental Science, 177, 4-7. https://doi.org/10.1192/bjp.177.1.4

Dein, S. (2013). Magic and Jinn among Bangladeshis in the United Kingdom Suffering from Physical and Mental Health Problems: Controlling the Uncontrollable. In Research in the Social Scientific Study of Religion, Volume 24 (pp. 193-219). Brill. https://doi.org/10.1163/9789004252073_009

Dein, S., Alexander, M., \& Napier, A. D. (2008). Jinn, Psychiatry and Contested Notions of Misfortune among East London Bangladeshis. Transcultural Psychiatry,45(1), 31-55. https://doi.org/10.1177/1363461507087997

Department of Health. (2005). Delivering race equality in mental health care: An action plan for reform inside and outside services and the Government's response to the independent inquiry into the death of David Bennett. Retrieved, 27 August 2018 from http://research.bmh.manchester.ac.uk/ReACH/resources/keypaper4. pdf

De Sousa, A. (2007). Religion, faith and psychiatry (A review). Journal of Pakistan Psychiatric Society, 1, 12-13.

Durà-Vilà, G., Hagger, M., Dein, S., \& Leavey, G. (2011). Ethnicity, religion and clinical practice: a qualitative study of beliefs and attitudes of psychiatrists in the United Kingdom. Mental Health, Religion \& Culture, 14(1), 53-64. https://doi.org/10.108 $0 / 13674676.2010 .495111$

Fazil, Q., \& Cochrane, R. (2003). The prevalence of depression in Pakistani women living in the West Midlands. Pakistani Journal of Women's Studies, 10, 21-30.

Gilbert, P., Gilbert, J., \& Sanghera, J. (2004). A focus group exploration of the impact of izzat, shame, subordination and entrapment on mental health and service use in South Asian women living in Derby. Mental Health, Religion \& Culture, 7, 109130. https://doi.org/10.1080/13674670310001602418

Haddad, Y. Y., \& Lummis, A. T. (1987). Islamic values in the United States: A comparative study. New York u.a.: Oxford University Press.

Hankir, A., Khalil, S., Wadood, Q., Madarbukus, D., Arifah Yunus, H., \& Bibi, S. et al. (2017). The Federation of Student Islamic Societies Programme to Challenge Mental Health Stigma In Muslim Communities in England: The FOSIS Birmingham Study. Psychiatria Danuba, 29(3), 512-520.

Hill, D. J., \& Bale, R. M. (1980). Development of the Mental Health Locus of Control and Mental Health Locus of Origin scales. Journal of Personality Assessment, 44(2), 148-156. https://doi.org/10.1207/s15327752jpa4402_5

Hussain, F. (2009). The Mental Health of Muslims in Britain. International Journal of Mental Health, 38(2), 21-36. https://doi.org/10.2753/IMH0020-7411380202

Jorm, A., Korten, A., Jacomb, P., Christensen, H., Rodgers, B., \& Pollitt, P. (1997). "Mental health literacy": a survey of the public's ability to recognise mental disorders and their beliefs about the effectiveness of treatment. The Medical Journal Of Australia, 166, 182 - 186. https://doi.org/10.5694/j.1326-5377.1997.tb140071.x

Keating, F., \& Robertson, D. (2004). Fear, black people and mental illness: A vicious circle? Health \& Social Care in the Community, 12(5), 439-447. https://doi. org/10.1111/j.1365-2524.2004.00506.x

Khalifa, N., Hardie, T., Latif, S., Jamil, I., \& Walker, D. (2011). Beliefs about Jinn, black magic and the evil eye among Muslims: Age, gender and first language influences. 
International Journal of Culture and Mental Health,4(1), 68-77. https://doi.org/10. $1080 / 17542863.2010 .503051$

Kunst, J., Sam, D., \& Ulleberg, P. (2013). Perceived islamophobia: Scale development and validation. International Journal Of Intercultural Relations, 37(2), 225-237. https://doi.org/10.1016/j.ijintrel.2012.11.001

Larson, D. B., Hohmann, A. A., Kessler, L. G., Meador, K. G., Boyd, J. H., \& Mcsherry, E. (1988). The Couch and the Cloth: The Need for Linkage. Psychiatric Services,39(10), 1064-1069. https://doi.org/10.1176/ps.39.10.1064

Leavey, G. (2010). The Appreciation of the Spiritual in Mental Illness: A Qualitative Study of Beliefs Among Clergy in the UK. Transcultural Psychiatry,47(4), 571-590. https://doi.org/10.1177/1363461510383200

Leavey, G. (2008). U.K. Clergy and People in Mental Distress: Community and Patterns of Pastoral Care. Transcultural Psychiatry,45(1), 79-104. https://doi. org/10.1177/1363461507087999

Lynch, E., \& Medin, D. (2006). Explanatory models of illness: A study of within-culture variation. Cognitive Psychology, 53(4), 285-309. https://doi.org/10.1016/j.cogpsych.2006.02.001

Maqsood, R. W. (2008). Islam. London: Collins.

McCabe, R., \& Priebe, S. (2004). Explanatory models of illness in schizophrenia: Comparison of four ethnic groups. British Journal Of Psychiatry, 185(01), 25-30. https:// doi.org/10.1192/bjp.185.1.25

Mir, G., Meer, S., Cottrell, D., McMillan, D., House, A., \& Kanter, J. (2015). Adapted behavioural activation for the treatment of depression in Muslims. Journal of Affective Disorders, 180, 190-199. https://doi.org/10.1016/j.jad.2015.03.060

Nickels, H., Thomas, L., Hickman, M., \& Silvestri, S. (2012). De-constructing "Suspect" Communities. Journalism Studies, 13(3), 340-355. https://doi.org/10.1080/146167 0X.2011.616412

NIMHE (2004) Celebrating our Cultures: Guidelines for Mental Health Promotion with the South Asian Community, Department of Health, London

Office for National Statistics (2013). Full story: What does the Census tell us about religion in 2011? Retrieved, 27 August 2018, from https://www.ons.gov.uk/peoplepopulationandcommunity/culturalidentity/religion/articles/fullstorywhatdoesthecensustellusaboutreligionin2011/2013-05-16

Penn, D. L., Guynan, K., Daily, T., Spaulding, W. D., Garbin, C. P., \& Sullivan, M. (1994). Dispelling the Stigma of Schizophrenia: What Sort of Information Is Best? Schizophrenia Bulletin,20(3), 567-578. https://doi.org/10.1093/schbul/20.3.567

Pescosolido, B., Martin, J., Long, J., Medina, T., Phelan, J., \& Link, B. (2010). “A disease like any other"? A decade of change in public reactions to schizophrenia, depression, and alcohol dependence. The American Journal of Psychiatry, 167(11), 132130. https://doi.org/10.1176/appi.ajp.2010.09121743

Piedmont, E. B. (1968). Referrals and Reciprocity: Psychiatrists, General Practitioners, and Clergymen. Journal of Health and Social Behavior, 9(1), 29. https://doi. org $/ 10.2307 / 2948315$

Pilkington, A., Msetfi, R. M., \& Watson, R. (2012). Factors affecting intention to access psychological services amongst British Muslims of South Asian origin. Mental Health, Religion \& Culture, 15(1), 1-22. https://doi.org/10.1080/13674676.2010.54 5947

Rao, D., Feinglass, J., \& Corrigan, P. (2007). Racial and ethnic disparities in mental illness stigma. Journal of Nervous and Mental Disease, 195, 1020-1023. https://doi. org/10.1097/NMD.0b013e31815c046e 
Rashid, S., Copello, A., \& Birchwood, M. (2012). Muslim faith healers' views on substance misuse and psychosis. Mental Health, Religion \& Culture, 15(6), 653-673. https://doi.org/10.1080/13674676.2011.613072

Rethink. (2007). Our voice: The Pakistani community's view of mental health and mental health services in Birmingham. Birmingham: Rethink.

Sheikh, A. (2007). Should Muslims have faith-based health services? British Medical Journal, 334, 74. https://doi.org/10.1136/bmj.39072.347720.68

Sheikh, A., \& Gatrad, A. (2009). Caring for Muslim patients. Oxford: Radcliffe.

Sheikh, S., \& Furnham, A. (2000). A cross cultural study of mental health beliefs and attitudes towards seeking help. Social Psychiatry and Psychiatric Epidemiology, 35, 326-334. https://doi.org/10.1007/s001270050246

Sheridan, L. (2006). Islamophobia Pre- and Post-September 11th, 2001. Journal of Interpersonal Violence, 21(3), 317-336. https://doi.org/10.1177/0886260505282885

Siddiqui, S. (2004) A Professional Guide for Canadian Imams. Winnipeg, MB, Canada: Islamic Social Services Association Inc.

Singh, S. P., Brown, L., Winsper, C., Gajwani, R., Islam, Z., Jasani, R., . . . Birchwood, M. (2015). Ethnicity and pathways to care during first episode psychosis: The role of cultural illness attributions. BMC Psychiatry,15(1). https://doi.org/10.1186/ s12888-015-0665-9

Steadman, H., Mulvey, E., Monahan, J., Robbins, P., Appelbaum, P., \& Grisso, T. et al. (1998). Violence by People Discharged From Acute Psychiatric Inpatient Facilities and by Others in the Same Neighborhoods. Archives Of General Psychiatry, 55(5), 393. https://doi.org/10.1001/archpsyc.55.5.393

Tabassum, R., Macaskill, A., \& Ahmad, I. (2000). Attitudes towards mental health in an urban Pakistani community in the United Kingdom. International Journal of Social Psychiatry, 46, 170-181. https://doi.org/10.1177/002076400004600303

VandeCreek, L., \& Lucas, A. M. (Eds.). (2001). The discipline for pastoral care giving: Foundations for outcome oriented chaplaincy. Binghamton, NY: Haworth Press.

Wallace, C., Mullen, P., \& Burgess, P. (2004). Criminal Offending in Schizophrenia Over a 25-Year Period Marked by Deinstitutionalization and Increasing Prevalence of Comorbid Substance Use Disorders. American Journal Of Psychiatry, 161(4), 716727. https://doi.org/10.1176/appi.ajp.161.4.716

Watts, S., Murray, C., \& Pilkington, A. (2013). Understanding and supporting psychological wellbeing: An exploration of the experiences of Islamic scholars. Mental Health, Religion \& Culture,17(4), 365-378. https://doi.org/10.1080/13674676.201 3.808177

Weatherhead, S., \& Daiches, A. (2010). Muslim views on mental health and psychotherapy. Psychology and Psychotherapy: Theory, Research and Practice, 83(1), 7589. https://doi.org/10.1348/147608309X467807

Williams, P. E., Turpin, G., \& Hardy, G. (2006). Clinical psychology service provision and ethnic diversity within the UK: A review of the literature. Clinical Psychology \& Psychotherapy, 13(5), 324-338. https://doi.org/10.1002/cpp.497

York, C., Perez-Chisti, A., Lewis, C., \& Yucel, S. (2011). The Effects of Ruqya on a NonMuslim: A Multiple Case Study Exploration (Doctor of Philosophy in Psychology). Institute of Transpersonal Psychology.

Young, J. L., Griffith, E. E., \& Williams, D. R. (2003). The Integral Role of Pastoral Counseling by African-American Clergy in Community Mental Health. Psychiatric Services, 54(5), 688-692. https://doi.org/10.1176/appi.ps.54.5.688 